\title{
Optimal Allocation of DGs with network reconfiguration using Improved Spotted Hyena Algorithm
}

\author{
ADEL A. ABO EL-ELA \\ Electrical Engineering Department \\ Faculty of Engineering, Menoufiya University \\ Shebin El-Kom, EGYPT
}

\author{
ABDULLAH M. SHAHEEN \\ Electrical Engineering Department \\ Faculty of Engineering, Suez University Suez, \\ EGYPT
}

\author{
RAGAB A. EL-SEHIEMY Electrical \\ Engineering Department Kafrelsheikh \\ University Kafrelsheikh, EGYPT
}

\author{
NORA K. EL-AYAA \\ High institue of Engineering and Technology, \\ El-Bajour, EGYPT
}

\begin{abstract}
Several studies of distribution network enhancement focused only on the optimization of either the integration of distributed generations (DGs) or system reconfiguration (SRC). However, very few researches have been exerted for incorporating both where this merge represents further complexities in searching for maximizing their benefits and minimizing the investments. This paper sheds light on the simultaneous allocation of DGs with SRC in distribution systems. It suggests a multi-objective formulation considering multiple objectives: active power loss minimization, total cost reduction, and voltage profile improvement. For solving this problem, an improved spotted hyena optimizer (ISHO) is developed. The SHO demonstrates a good feature in finding the optimal or nearly optimal subset with simple structure to minimize the given fitness function. To support the exploration characteristics of the proposed ISHO, it augments a switch strategy in the updating mechanism of the spotted hyena's positions and random positions are generated instead of the violated spotted hyenas. The simulations are investigated for the integration of a single and multiple DGs with SRC for two standard systems, which are IEEE 33 and 69-node test systems. The obtained results prove the efficiency of the suggested method for the network manager to find the optimal DGs allocation with SRC considering multiple criteria. These results are compared with previous literatures to demonstrate the effectiveness of the proposed algorithm.
\end{abstract}

Key-Words: - Distribution networks, distribution generation, system reconfiguration, spotted hyena optimization.

Received: September 25, 2019. Revised: April 11, 2020. Accepted: April 17, 2020. Published: April 30, 2020.

\section{Introduction}

In electrical distribution companies, there are continuous modernization all over the world for the sake of better enhancement the performance of the distribution networks. In order to do this, there are various recent studies like system reconfiguration (SRC) [1], voltage regulators [2], power electronic devices of soft open points [3], storage sources [4], CBs allocations $[5,6]$, DGs allocation $[7,8]$, ..., etc,.

With the rising utilizations of distribution automation with their suitable allocations of remote terminal units, real-time system reconfiguration (SRC) has become very efficient tool for controlling and operating of the distribution networks. SRC is an optimization problem which is considered a highly nonlinear, mixed integer type and nondifferentiable [9]. This is due to the discrete nature of the switches and the variations in the load and the generation from renewables DGs, e.g. the wind and solar. However, the system reconfiguration reduces the system losses, it may not be sufficient to meet the increasing demand. At higher loading levels, during peak, SRC alone may not be enough to enhance the distribution networks [10].

The implementation of DGs performs a major support for acquiring great benefits in low, medium and high voltage power networks, like, decreasing the overall losses, reducing the loading of the branches, and improving the power quality indicators $[11,12]$. On the other side, the high costs of DGs, related to their investment and maintenance, are considerable barriers for realizing the desired goals. Thus, Installation of the DGs must be at proper sizes and locations for maximizing their expected benefits. In [13], genetic algorithm has been utilized for locating and sizing the DGs in radial distribution networks where the loss sensitivity factor (LSF) has been applied to reduce the DGs search locations. In [14], an analytical method has been suggested based on LSF and 
exhaustive power flow. However, in those references $[13,14]$, the minimization of the active losses has been taken as a mono-target. In [15], renewable DGs placement and sizing have been integrated in a mixed-integer nonlinear problem for improving the voltage stability but it has been taken as a mono-target as well.

In [16], binary particle swarm optimization (BPSO) has been introduced for DGs installation for decreasing the power losses. In [17], a modified optimizer based on bacterial foraging has been performed presented for minimizing an objective of the total power losses. In [18], a multi-objective method has been carried out for economic analysis of DGs owner's and distribution company's point, but the simulation study has been applied on small IEEE 33-node test system.

There are some works in literature about simultaneous optimization of SRC and DGs installation. In [19] and [20], hyper cube ant colony algorithm and modified plant growth optimization have been applied for this simultaneous implementation, but they have been only executed for a small-scale system such as 33 bus system. In [21], the power losses minimization and node voltage deviations have been treated through harmony search algorithm where the optimal DGs locations have been sensitivity analyzed to identify their candidate locations.

Recently, spotted hyena optimization (SHO) has been developed from the inspiration of the behavior of the spotted hyena in the nature. They are searching for the optimal way for hunting preys in an innovative way. SHO is simple, flexible, and easy to use with very few parameters. This paper employs an application of this simple optimizer for simultaneous allocation of DGs with SRC in distribution systems. It suggests a multi-objective formulation considering multiple objectives: active power loss minimization, total cost reduction, and voltage profile improvement. Added to that, an improved spotted hyena optimizer (ISHO) is developed to support the exploration characteristics of the proposed ISHO. This proposed ISHO augments a switch strategy in the updating mechanism of the spotted hyena's positions and random positions are generated instead of the violated spotted hyenas. The simulations are investigated for the integration of a single and multiple DGs with SRC for two standard systems, which are IEEE 33 and 69-node test systems. The obtained results prove the efficiency of the suggested method for the network manager to find the optimal DGs allocation with SRC considering multiple criteria. These results are compared with previous literatures to demonstrate the effectiveness of the proposed algorithm.

\section{Problem Formulation \\ 2.1 Objective functions}

\subsubsection{Minimization of active power loss}

The first considered objective is to minimize the sum of power losses in all distribution branches, which can be defined as:

$$
P_{\text {losses }}=\sum_{b=1}^{N_{b}} R_{b} I_{b}^{2}
$$

where, $P_{\text {losses }}$ refers to active power loss in the branches, $N_{b}$ are the set of branches, $I_{b}$ is the current flow in the branch $b$.

\subsubsection{Minimization of Total Costs ( $\$ /$ Year)}

The second objective is to minimize the total costs which are related to the operational costs of the power losses, the investment costs on DGs installations and the purchased costs from the grid. This objective function can be presented as follows:

$T_{C}=k_{e 1} * D * P_{\text {losses }}+k_{d g} * C_{r f} *$

$\sum_{i=1}^{N_{d g}} P_{d g_{i}}+\quad K_{e 2} * D * S_{\text {grid }}$

Where, $T_{C}$ is the total costs; $k_{e 1}$ is the operational cost for the hourly power ( $\$ / \mathrm{Mwhr})$; $D$ is the duration hours in the year; $k_{d g}$ is the operational and maintenance cost for DGs $(\$ / \mathrm{kw}) ; C_{r f}$ is the cost recovery factor of DG; $N_{d g}$ is the number of DGs; $P_{d g}$ is the power of DGs; $K_{e 2}$ is the $\$ / M w h r$ energy price; $S_{\text {grid }}$ is the absorbed power from the grid (Mw).

\subsection{Constraints}

The optimal simultaneous reconfiguration and DGs allocation in the distribution network must be respect to certain security and topological constraints as follows:

\subsubsection{Equality constraints}

The power balance constraints can be defined as follows:

$P_{i}^{i n j}=\sum_{k=1}^{n}\left|V_{i}\right|\left|V_{k}\right|\left(G_{i k} \cos \left(\Theta_{i k}\right)+\right.$ $\left.B_{i k} \sin \left(\Theta_{i k}\right)\right)$

$Q_{i}^{i n j}=\sum_{k=1}^{n}\left|V_{i}\right|\left|V_{k}\right|\left(G_{i k} \sin \left(\Theta_{i k}\right)-\right.$ $\left.B_{i k} \cos \left(\Theta_{i k}\right)\right)$

\subsubsection{Inequality constraints Bus voltage limits$$
V^{\min } \leq V_{i} \leq V^{\max }
$$ 
Where, $V_{i}$ is the voltage at each bus $i$ should be kept between its minimum and maximum values as the superscripts, 'min' and 'max', indicate, respectively.

\section{Branch current limits}

$$
\left|I_{b}\right| \leq I_{b}^{\max }
$$

The magnitude of the current $I_{b}$ at each branch should not exceed its maximum thermal value $I_{b}^{\max }$.

\section{DGs capacity constraint}

Also, the DGs sizes $\left(P_{d g}\right)$ is bounded by their maximum limit as:

$$
0 \leq P_{d g j} \leq P_{d g j}^{\max } \quad j=1,2, \ldots . N_{d g}
$$

\section{DGs penetration Level}

In general, the penetration rate of DGs according to the renewable energy policies of countries, it is assumed that the total injected capacity of the DGs should be less than $75 \%$ of the active power load in the distribution networks; i.e.:

$$
\sum_{i=1}^{N_{d g}} P_{d g_{i}} \leq 0.75 * P_{\text {load }}
$$

\section{Topological constraint}

The distribution network reconfiguration during the evolutionary process should be operated in radial topology. There must be no loops in the network and all loads have to be supplied. To include this constraint, it can be formulated as follows:

$$
\begin{gathered}
\operatorname{det}(A)=1 \text { or }-1 \quad \text { (radialsystem }) \\
\operatorname{det}(A)=0(\text { notradial })
\end{gathered}
$$

where $A$ is the branch-bus incidence matrix [23].

\section{Proposed Algorithm for Solving the Reconfiguration Problem}

\subsection{Spotted Hyena Algorithm}

In SHA [22], the spotted hyenas update their positions respect to the target solution. This behavior is done as:

$$
\begin{aligned}
& \vec{D}_{\mathrm{SH}}=\left|\vec{C} \vec{X}_{T}-\vec{X}_{\mathrm{SH}}\right| \\
& \vec{X}_{\mathrm{SH}}=\vec{X}_{T}-\vec{A} \vec{D}_{\mathrm{SH}}
\end{aligned}
$$

where $D_{\mathrm{SH}}$ represents the remoteness between the spotted hyena's position $\left(\mathrm{X}_{\mathrm{SH}}\right)$ and the target position of the prey $\left(\mathrm{X}_{\mathrm{T}}\right)$. $\mathrm{A}$ and $\mathrm{C}$ are the updated co-efficient parameters as follows:

$$
\begin{aligned}
& \vec{A}=2 \vec{a} r-\vec{a} \\
& \vec{C}=2 \mathrm{r}
\end{aligned}
$$

where, the vector a, that symbolized in Eq. (3), is linearly decreased from 5 to 0 as:

$$
\vec{a}=5 .\left(1-\frac{\text { iter }}{\text { Max }_{\text {iter }}}\right)
$$

where, $r$ is a random number. Then, a specified group of a number $(\mathrm{N})$ of the best hyenas $\left(\mathrm{C}_{\mathrm{H}}\right)$ is consisted as follows:

$\vec{C}_{H}=\vec{X}_{\mathrm{SH}}+X_{\mathrm{SH}+1}+X_{\mathrm{SH}+2}+\ldots \ldots . . X_{\mathrm{SH}+N}$

where, $\mathrm{N}$ refers to a specified number of the hyenas, which is evaluated as:

$$
\begin{aligned}
& N=\operatorname{count}_{\mathrm{n} 1} \\
& \vec{M})
\end{aligned}
$$

where, $\mathrm{n} 1$ is the number of the spotted hyenas. $\mathrm{M}$ is a random vector within range $[0.5$, 1]. Afterwards, the hyenas update their positions in order to attack their tracked preys via Eq. (18) as:

$$
\vec{X}_{\text {SHnew }}=\frac{\vec{C}_{H}}{N}
$$

After updating the positions of the spotted hyenas, they are checked related to their bounds as follows:

$$
\begin{aligned}
& X_{\text {SHnew }}(d)= \\
& \left\{\begin{array}{c}
X(d)^{\max } \text { if } X_{\text {SHnew }}(d)>X(d)^{\max } \\
X_{\text {SHnew }}(d) \text { if } X(d)^{\text {min }}>X_{\text {SHnew }}(d)>X(d)^{\max } \\
X(d)^{\text {min }} \text { if } X_{\text {SHnew }}(d)<X(d)^{\text {min }} \\
d=1,2, \ldots \ldots \ldots . \text { Dim }
\end{array}\right.
\end{aligned}
$$

Where, the superscripts 'min' and 'max' indicate the lower and upper limits. Dim refers to the considered dimension.

\subsection{Improved Spotted Hyena Algorithm (ISHA) for the considered problem}

In the basic SHA, the group of the best hyenas $\left(\vec{C}_{H}\right)$ should be selected at each iteration. An important question rises here is What if there is no valid solution in the initial population? This may be occurred if the initial hyenas don't get convergence in the Load Flow 
or some of the loads aren't supplied. This will cause an empty group of the best hyenas $\left(\vec{C}_{H}\right)$ and will block the updating mechanism of the SHA. A first improvement is proposed to avoid this block by applying a switch strategy to update the spotted hyena's positions as follows:

$X_{\text {SHnew }}=\overrightarrow{X_{\mathrm{SH}}}+\varepsilon \vec{R}\left(1-\frac{\text { iter }}{\text { Max }_{\text {iter }}}\right)$

Where, $\varepsilon$ is very small value $(\varepsilon=0.001)$ and $\mathrm{R}$ is a random vector within range $[0.5,1]$. Another modification is proposed to support the exploration characteristics for searching for new areas. This improvement is to generate new random positions to the violated spotted hyenas as follows:

$$
\begin{aligned}
& X_{\text {SHnew }}(d)= \\
& \left\{\begin{array}{c}
X(d)^{\text {max }} \text { if } \mathrm{X}_{\mathrm{SHnew}}(d)>X(d)^{\max } \\
X_{\mathrm{SH}}{ }^{*}(d) \text { if } \mathrm{X}(d)^{\text {min }}>X_{\mathrm{SHnew}}(d)>X(d)^{\max } \\
X(d)^{\text {min }} \text { if } \mathrm{X}_{\mathrm{SHnew}}(d)<X(d)^{\text {min }} \\
\quad d=1,2, \ldots \ldots \ldots . . \text { Dim }
\end{array}\right.
\end{aligned}
$$

Where, $\mathrm{XSH}_{\mathrm{SH}^{*}}(\mathrm{~d})$ is a random value within the permissible range for each control variable (d). Fig.1 displays the flowchart of the proposed ISHA for handling the considered problem.

\section{Results and Comments}

The optimization problem of optimal simultaneous reconfiguration and DGs allocation in the distribution network is solved using the ISHA. The program is developed in the MATLAB software. In this study, three different cases are considered:

- Case 1: One DG $\left(N_{d g}=1\right)$

- Case 2: Two DGs $\left(N_{d g}=2\right)$

- Case 3: Three DGs $\left(N_{d g}=3\right)$

The simulation runs are performed using the proposed ISHO with number of spotted hyena $=$ 20, Max $_{\text {iter }}=200 . K_{d g}=5000 \$ / \mathrm{kW}, k_{e 1}=K_{e 2}=$ $60 \$ / \mathrm{Mwhr}[24,25], D=8760 \mathrm{hr} ; C_{r f}$ is the cost recovery factor of DG, which can be calculated as:

$$
C_{r f}=\frac{I_{r}\left(1+I_{r}\right)^{\mathrm{Y}}}{\left(1+I_{r}\right)^{\mathrm{Y}}-1}
$$

where, $\mathrm{I}_{\mathrm{r}}$ represents the corresponding interest rate, while $\mathrm{Y}$ is the regarded $\mathrm{DG}$ lifetime.

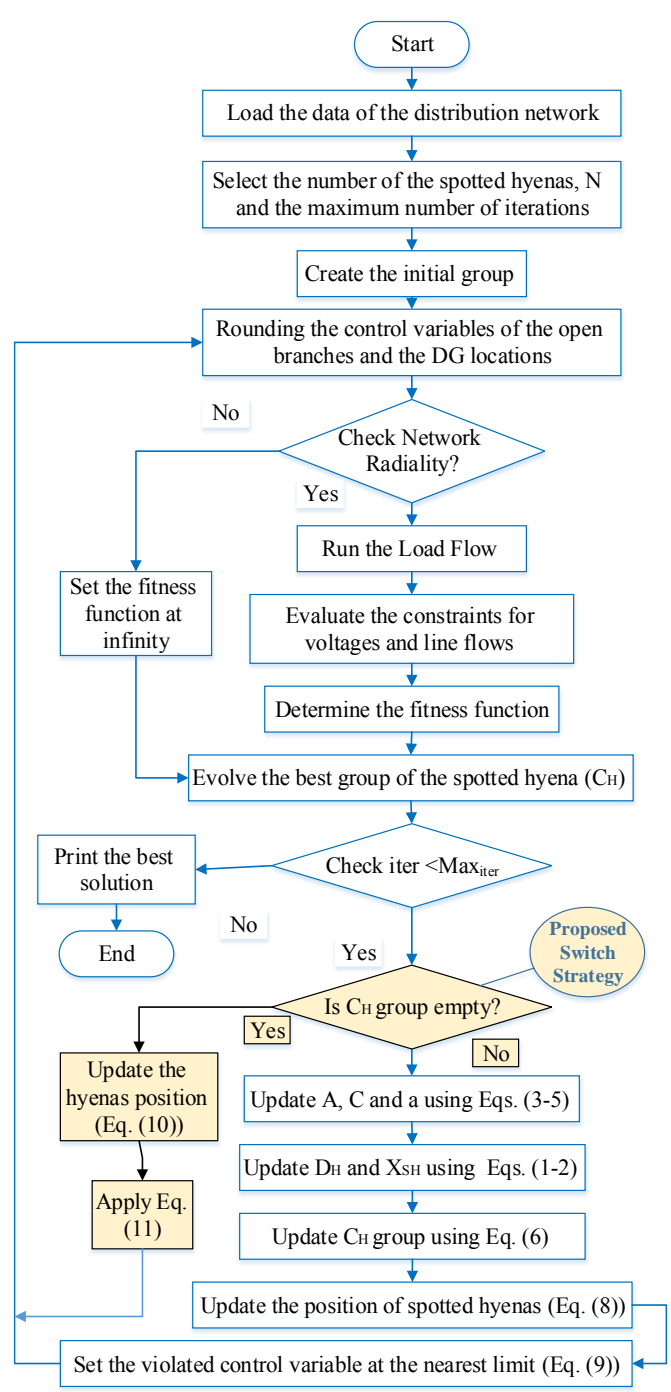

Fig. 1.

The phases of the proposed ISHA for handling the considered problem

\subsection{First Test System: IEEE 33-bus system}

The first system is IEEE 33-bus radial distribution system. The system is $12.6 \mathrm{KV}$ with 5 open branches from 33-37. The line and bus data are given in [26]. The proposed ISHO is applied for this system and the simulation results are depicted in Table 1 . The regarded convergence characteristics are described in Fig. 2 for the three cases studied. From both;

Huge reduction is acquired in the power losses, where the proposed ISHO reduces it with percentage of 53.88, 61.22 and 64.94 for the three cases, respectively. This huge reduction in the power losses is accompanied with high reduction in the total costs which are related to the operational costs of the power losses, the investment costs on DGs installations and the purchased costs from the grid. The maximum reduction, that acquired by 
the proposed ISHO, is recorded at the third case with reduction percentage of 13.76 compared to the initial case.

Good convergence characteristics are illustrated for the three cases as in Fig. 2.

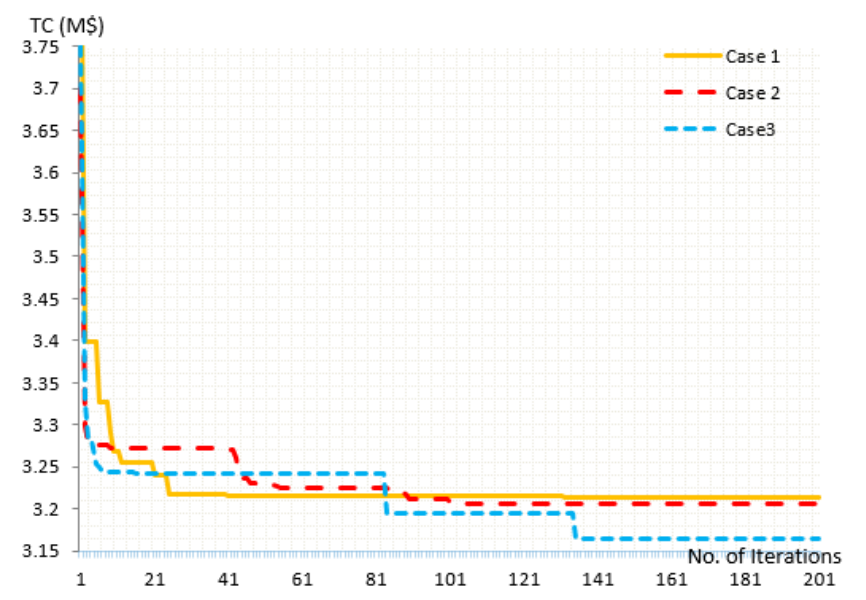

Fig. 2. Convergence curve of the proposed ISHO for the three cases

Significant enhancement of the node voltages is acquired, where the minimum obtained voltage via the proposed ISHO of $0.96,0.96$ and 0.964 for the three studied cases, respectively compared to 0.91 at the initial case.

The maximum enhancement of the node voltages, that acquired by the proposed ISHO, is recorded at the third case where all the node voltages are improved as displayed in Fig. 3.

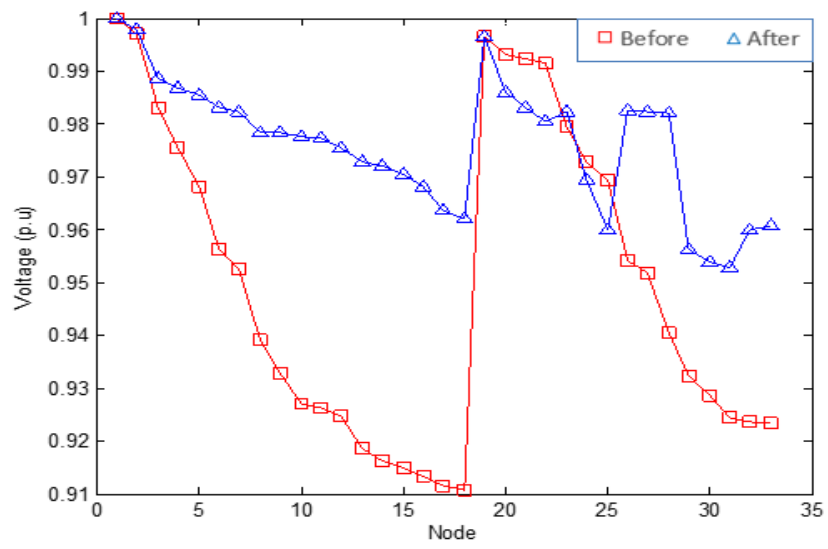

Fig. 3. Node voltages via the proposed ISHO for the third case

Table 1: Application of the proposed ISHO for the first test system

\begin{tabular}{|c|c|c|c|c|}
\hline & Initial & Case 1 & Case 2 & Case 3 \\
\hline Open branches & $33-37$ & $10,14,28,30 \& 33$ & $11,14,17,28 \& 33$ & $7,11,17,28 \& 34$ \\
\hline DG size (location) & - & $1149(18)$ & $870(31) 462(15)$ & $344(18), 850(29), 274(16)$ \\
\hline Losses (KW) & 208.5 & 96.14 & 80.86 & 73.11 \\
\hline Losses reduction (\%) & - & 53.88 & 61.22 & 64.94 \\
\hline Total cost (M\$) & - & 3.237 & 3.20 & 3.18 \\
\hline
\end{tabular}

Table 2: Comparative results for the first test system considering one DG

\begin{tabular}{|c|c|c|c|c|c|}
\hline & Open branches & DG size (location) & KW Losses & TC (M\$) & Reduction of TC (\%) \\
\hline BPSO & $7,11,30,35 \& 37$ & $1354(18)$ & 97.43 & 3.22 & 12.60 \\
\hline SHOA & $6,10,24,30 \& 34$ & $1418(15)$ & 114.10 & 3.24 & 12.12 \\
\hline DE1 & $9,13,19,28 \& 34$ & $1497(29)$ & 125.21 & 3.25 & 11.89 \\
\hline DE2 & $7,11,27,31 \& 34$ & $1181(16)$ & 105.5 & 3.25 & 12.09 \\
\hline ISHOA & \multicolumn{7}{|c|}{ Highest Reduction } & 13.03 \\
\hline
\end{tabular}

Table 3: Comparative results for the First Test System considering Two DGs

\begin{tabular}{|c|c|c|c|c|c|}
\hline & Open branches & DG size (location) & KW Losses & TC (M\$) & Reduction of TC (\%) \\
\hline BPSO & $7,8,9,17 \& 37$ & $541(18), 979(12)$ & 89.05 & 3.20 & 13.36 \\
\hline SHOA & $5,7,10,15 \& 34$ & $431(28), 973(33)$ & 96.01 & 3.22 & 12.72 \\
\hline DE1 & $10,17,33,34 \& 37$ & $871(33), 457(1)$ & 103.35 & 3.23 & 12.51 \\
\hline DE2 & $7,10,27,33 \& 36$ & $389(10), 1002(32)$ & 86.73 & 3.21 & 13.06 \\
\hline ISHOA & Highest Reduction & & 13.36 \\
\hline
\end{tabular}

Table 4: Comparative results for the First Test System considering Three DGs

\begin{tabular}{|c|c|c|c|c|c|}
\hline & Open branches & DG size (location) & KW Losses & TC (M\$) & Reduction of TC (\%) \\
\hline BPSO & $8,14,31,33 \& 37$ & $92(14) 658(16) 770(16)$ & 82.78 & 3.19 & 13.5 \\
\hline SHOA & $7,8,10,28 \& 31$ & $703(7) 551(17) 130(8)$ & 92.57 & 3.22 & 12.82 \\
\hline DE1 & $11,13,33,34 \& 37$ & $432(18), 1029(30)$ & 96.36 & 3.21 & 13.035 \\
\hline DE2 & $4,26,30,33 \& 34$ & $661(14) 721(7) 95(3)$ & 109.5 & 3.24 & 12.21 \\
\hline ISHOA & Highest Reduction & & 13.76 \\
\hline
\end{tabular}


Moreover, a comparative analysis is conducted between the proposed ISHO, the basic SHO, BPSO [27], two differential evaluation variants (DE1 [28]) and (DE2 [25]). This comparative analysis is carried out and tabulated for Case 1 in Table 2, Case 2 in Table 3 and Case 3 in Table 4, respectively. This analysis declares the importance of the proposed modifications in ISHO over the basic SHO. Not only that, but the proposed ISHO always finds the greatest reduction percentage for all the considered cases.

\subsection{Second Test System: IEEE 69-bus system}

The IEEE 69-bus distribution system has open branches from 69-73. The line and bus data for this system are given in [29]. The proposed ISHO is applied for this system and the simulation results for the three considered cases are depicted in Table 5. The regarded convergence characteristics are described in Fig. 4 for the three cases studied.
Added to that, a comparative analysis is conducted between the proposed ISHO and other optimizers as tabulated in Tables 6-8. This analysis declares that the proposed ISHO always finds the greatest reduction percentage for all the considered cases. Similar results are obtained.

Huge reduction is acquired in the power losses with percentages of 71.8, 70.43 and 75.24 for the three studied cases, respectively. This is accompanied with high reduction in the total costs. The maximum reduction is carried out at the third case with reduction percentage of 9.29 compared to the initial case.

Good convergence characteristics are illustrated for the three cases as in Fig. 4.

Significant enhancement of the node voltages is acquired, where the minimum voltage is improved for the three studied cases, respectively. As an example, Fig. 5 shows the node voltages via the proposed ISHO at the third case where all the node voltages are improved compared to the initial case.

Table 5: Application of the proposed ISHO for the second test system

\begin{tabular}{|c|c|c|c|c|}
\hline & Initial & Case 1 & Case 2 & Case 3 \\
\hline Open branches & $69-73$ & $8,10,12,20 \& 23$ & $8,10,12,20 \& 26$ & $8,10,12,14 \& 26$ \\
\hline DG size (location) & - & $2566(61)$ & $1044(62), 1790(59)$ & $1313(62), 23(46), 132(50)$ \\
\hline Losses (KW) & 225 & 63.44 & 66.52 & 55.72 \\
\hline Losses reduction (\%) & - & 71.8 & 70.43 & 75.24 \\
\hline Total cost (M\$) & - & 3.37 & 3.36 & 3.35 \\
\hline
\end{tabular}

Table 6: Comparative results for the second test system considering one DG

\begin{tabular}{|c|c|c|c|c|c|}
\hline & Open branches & DG size (location) & KW Losses & TC (M\$) & Reduction of TC (\%) \\
\hline BPSO & $16,25,42,45,57$ & $1520(27)$ & 97.37 & 3.472 & 6.27 \\
\hline SHOA & $20,26,42,58,71$ & $1306(30)$ & 138.24 & 3.55 & 4.09 \\
\hline DE1 & $8,9,13,20,22$ & $2498(27)$ & 157.87 & 3.50 & 5.47 \\
\hline DE2 & $20,40,54,62,69$ & $1971(12)$ & 110.0238 & 3.4728 & 6.26 \\
\hline ISHOA & \multicolumn{7}{|c|}{ Highest Reduction } \\
\hline
\end{tabular}

Table 7: Comparative results for the second test system considering Two DGs

\begin{tabular}{|c|c|c|c|c|c|}
\hline & Open branches & DG size (location) & KW Losses & TC (M\$) & Reduction of TC (\%) \\
\hline BPSO & $15,42,45,58,61$ & $656(12), 864(21)$ & 92.89 & 3.47 & 6.27 \\
\hline SHOA & $10,14,20,26,58$ & $66(27), 1454(46)$ & 117.602 & 3.51 & 5.07 \\
\hline DE1 & $8,10,14,17,23$ & $1042(26), 1361(18)$ & 111.405 & 3.45 & 6.84 \\
\hline DE2 & $12,15,40,62,72$ & $809(4), 1614(58)$ & 111.7 & 3.43 & 7.38 \\
\hline ISHOA & \multicolumn{7}{|c|}{ Highest Reduction } & 9.22 \\
\hline
\end{tabular}

Table 8: Comparative results for the second test system considering Three DGs

\begin{tabular}{|c|c|c|c|c|c|}
\hline & Open branches & DG size (location) & KW Losses & TC $(\mathrm{M} \$)$ & Reduction of TC $(\%)$ \\
\hline BPSO & $9,12,19,55,62$ & $0(18), 2(2), 1518(15)$ & 99.02 & 3.47 & 6.27 \\
\hline SHOA & $14,20,42,58,64$ & $116(27), 118(20), 121(23)$ & 116.21 & 3.51 & 5.07 \\
\hline DE1 & $4,9,11,19,23$ & $117(8), 1473(24), 696(31)$ & 119.21 & 3.45 & 6.84 \\
\hline DE2 & $40,43,56,61,70$ & $1365(50), 70(46), 1074(41)$ & 106.48 & 3.43 & 7.38 \\
\hline ISHOA & \multicolumn{7}{|c|}{ Highest Reduction } & 9.29 \\
\hline
\end{tabular}




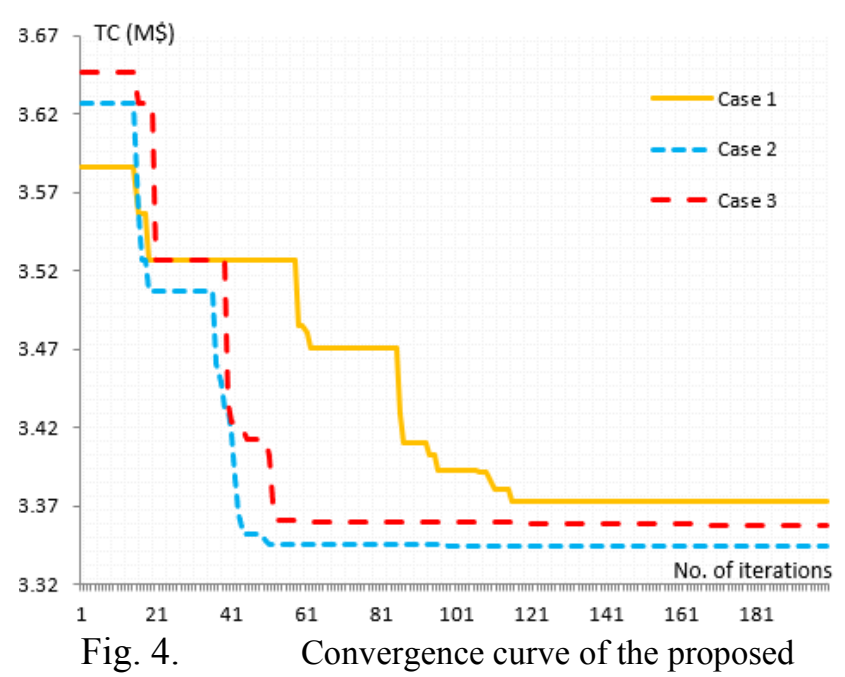

ISHO for the three cases for the second system

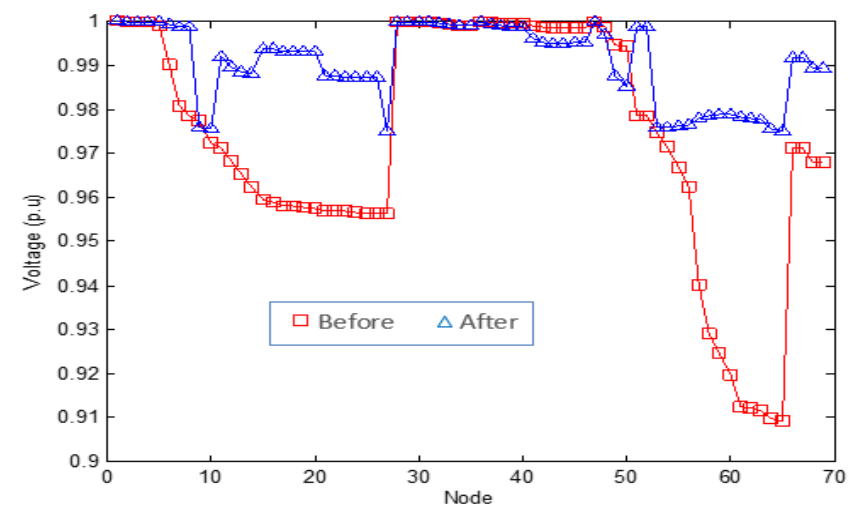

Fig. 5. Node voltages via the proposed ISHO for the third case for the second test system

\section{Conclusions}

In this paper, the incorporation of distributed generations (DGs) allocations and system reconfiguration (SRC) is has been successfully carried out considering multiple optimization criteria. The objective function includes power loss minimization, investment costs reduction, and purchased power decreasing, with respect to the system topology and security constraints. In this paper, an improved spotted hyena optimization (ISHO) is proposed by supplying a switch strategy in the updating mechanism of the spotted hyena's positions whilst random positions are generated instead of the violated spotted hyenas. The simulations are investigated for the integration of a single and multiple DGs with SRC for two standard systems, which are IEEE 33 and 69-node test systems. The obtained results via the suggested ISHO are compared to the basic SHO, BPSO, and DE to find the optimal DGs allocation with
SRC. The obtained results demonstrate the effectiveness of the proposed ISHO with highest reduction in the total costs related to the operational costs of the power losses, the investment costs on DGs installations and the purchased costs from the grid. It provides good convergence characteristics and significant enhancement of the node voltages where the minimum obtained voltage via the proposed ISHO is improved for all the studied cases.

\section{References:}

[1] A.M. Shaheen and R.A. El-Sehiemy, "A Multiobjective Salp Optimization Algorithm for Techno-Economic-Based Performance Enhancement of Distribution Networks", IEEE Systems Journal, 2020.

[2] A.M. Elsayed, M.M. Mishref and S.M. Farrag, "Distribution system performance enhancement (Egyptian distribution system real case study)", Int. Trans. Elect. Energy Syst. 2018.

[3] M.B. Shafik, H. Chen, G.I. Rashed, R.A. ElSehiemy, M.R. Elkadeem, S. Wang, "Adequate topology for efficient energy resources utilization of active distribution networks equipped with soft open points," IEEE Access, vol. 7, pp. 99003-99016, 2019.

[4] S. Bahceci, A. Dogan, T. Yalcinoz, F. Daldaban, "Energy storage system location selection for smart grid applications on distribution networks", Electrical Engineering, vol. 99, pp. 357-366, 2017.

[5] A.M. Shaheen, R. El Sehiemy, and S.M. Farrag, "Adequate planning of shunt power capacitors involving transformer capacity release benefit", IEEE Sys. J., vol. 12, no. 1, pp. 373-382, March 2018.

[6] A.M. Shaheen and R.A. El-Sehiemy, "Optimal allocation of capacitor devices on MV distribution networks using crow search algorithm", CIRED-Open Access Proceedings Journal, vol. 1, pp. 2453-2457, 2017.

[7] A.A. Abou El-Ela, R.A. El-Sehiemy and A.S. Abbas, "Optimal placement and sizing of distributed generation and capacitor banks in distribution systems using water cycle algorithm", IEEE Syst. J., vol. 12, no. 4, pp. 3629-3636, 2018.

[8] A.A. El-Ela, R. El Sehiemy, A.M. Shaheen, and I.A. Eissa, "Optimal allocation of DGs and capacitor devices using improved grey wolf optimizer", 21st International Middle 
East Power Systems Conference (MEPCON'21), Tanta University, 2019.

[9] S. Mishra, D. Das, S. Paul, "A comprehensive review on power distribution network reconfiguration", Energy Syst., vol. 8, pp. 227-284, 2017.

[10] I. B. Hamida, S. B. Salah, F. Msahli, M. F. Mimouni, "Optimal network reconfiguration and renewable DG integration considering time sequence variation in load and DGs", Renewable Energy, vol. 121, pp. 66-80, 2018.

[11] A. Bayat and A. Bagheri, "Optimal active and reactive power allocation in distribution networks using a novel heuristic approach" Applied Energy, vol. 233-234, pp. 71-85, 2019.

[12] G. Gharehpetian, and S. Agah, "Distributed generation systems, design, operation and grid integration", Elsevier, 2017.

[13] T.N. Shukla, S.P. Singh, V. Shrinivasarao, K.B. Naik, "Optimal sizing of distributed generation placed on radial distribution systems", Elect. Power Compon. Syst., vol. 38, pp. 260-274, 2010.

[14] D.Q. Hung, N. Mithulananthan, "Multiple distributed generators placement in primary distribution networks for loss reduction", IEEE Trans. Ind. Electron., vol. 60, pp. 17001708, 2013.

[15] R.S. Al Abri, E. F. El-Saadany, Y. M. Atwa, "Optimal placement and sizing method to improve the voltage stability margin in a distribution system using distributed generation", IEEE Trans. Power Syst., vol. 28, pp. 326-334, 2013.

[16] S. Kansal, K. Vishal, T. Barjeev, "Optimal placement of different type of DG sources in distribution networks", Int. J. Electr. Power Eng. Syst., vol. 53, pp. 752-760, 2013.

[17] S. Devi and M. Geethanjali, "Application of Modified Bacterial Foraging Optimization Algorithm for Optimal Placement and Sizing of Distributed Generation", Expert Syst. Applications, vol. 41, no. 6, pp. 2772-2781, May 2014.

[18] A. Ameli, S. Bahrami, F. Khazaeli, M. R. Haghifam, "A multiobjective particle swarm optimization for sizing and placement of DGs from DG owner's and distribution company's viewpoints", IEEE Trans. Power Deliv., vol. 29, pp. 1831-1840, 2014.

[19] N. Ranjan, "Optimal feeder reconfiguration of distributed system with distributed generation units using Hypercube-Ant Colony optimization algorithm", Int. J. Electr. Eng. Inform., vol. 6, pp. 107-128, 2014.

[20] R. Rajaram, K.S. Kumar, N. Rajasekar, "Power system reconfiguration in a radial distribution network for reducing losses and to improve voltage profile using modified plant growth simulation algorithm with Distributed Generation (DG)", Energy Reports, vol. 1, pp. 116-122, 2015.

[21] R.S. Rao, K. Ravindra, K. Satish, S.V. Narasimham, "Power loss minimization distribution system using network reconfiguration in the presence of distributed generation", IEEE Trans. Power Syst., vol. 28, pp. 317-325, 2013.

[22] G. Dhiman, V.Kumar, "Spotted hyena optimizer: a novel bio-inspired based metaheuristic technique for engineering applications", Adv. Eng. Softw. 114 (Supplement C), 48-70 (2017).

[23] J.J. Grainger, W.D. Stevenson, "Power system analysis", Mcgraw-Hill Book Company, New York, 1994.

[24] A.M. Shaheen, R. El Sehiemy, and S.M. Farrag, "A Novel Adequate Bi-Level Reactive Power Planning Strategy", Int. J. Elect. Power Energy Syst., vol. 78, pp. 897-909, 2016.

[25] A.M. Shaheen and R. A. El-Sehiemy, "A reactive power planning procedure considering iterative identification of VAR candidate buses", Neural Computing and Applications, June 2017.

[26] M.A. Kashem, V. Ganapathy, G.B. Jasmon, and M.I. Buhari, "A novel method for loss minimization in distribution networks", Int. Conf. electric utility deregulation restructuring power tech., pp. 251-257, 2000.

[27] A.A. El-Ela, R. El Sehiemy, and N. El-Ayaat, "Multi-objective Binary Particle Swarm Optimization Algorithm for Optimal Distribution System reconfiguration", 21st Int. Middle East Power Systems Conf. (MEPCON'21), Tanta University, 2019.

[28] A.M. Shaheen, R. El Sehiemy, and S. M. Farrag, "MOPF solution methodology", IET Gen. Transm. Dist., vol.11, no.2, 2017.

[29] M.E. Baran, and F.F. Wu. "Optimum sizing of capacitor placed on radial distribution systems", IEEE Trans PWRD 1989. pp: 735743. 